06

\title{
Развитие метода измерения диффузионного сопротивления структур материалов пористых электродов на основе протонпроводящего иономера и углеродных наноматериалов
}

\author{
(C) Н.В. Глебова, А.О. Краснова, А.А. Томасов, Н.К. Зеленина, А.А. Нечитайлов \\ Физико-технический институт им. А.Ф. Иоффре РАН, \\ 194021 Санкт-Петербург, Россия \\ ฯ e-mail: aan.shuv@mail.ioffe.ru
}

(Поступило в Редакцию 26 января 2017 г.)

\begin{abstract}
Развит метод измерения сопротивления массовому транспорту кислорода пористых электродов на основе микро- и наноструктур протонпроводящего иономера Nafion, $\mathrm{Pt} / \mathrm{C}$, углеродных нановолокон со сверхвысокой пористостью. Метод основан на измерении предельной плотности тока электровосстановления кислорода при контролируемом потоке кислорода через электрохимическую систему и вычислении сопротивления массовому транспорту кислорода с использованием соотношения, основанного на законе Фика. Метод применен к изучению массотранспортных потерь электрода мембранно-электродного блока (МЭБ) и электродного материала на дисковом вращающемся электроде и может быть использован при разработке новых электродов.
\end{abstract}

DOI: 10.21883/JTF.2017.12.45211.2183

\section{Введение}

Транспортные свойства пористых электродов электрохимических преобразователей энергии (электролизеры воды, низкотемпературные топливные элементы с полимерной мембраной), как известно, оказывают весьма существенное влияние на их эффективность. Современные же требования к таким устройствам возрастают по мере внедрения их в экономику передовых стран мира. Электроды таких устройств состоят из компонентов с различными функциональными свойствами, обычно это электрохимически активный компонент (платина) на носителе (углеродная сажа) и компонент, обеспечивающий ионный (протонный) транспорт (иономер Nafion). В некоторых случаях используют гидрофобизирующие и структурирующие добавки. Исследование массового транспорта в подобных пористых электродах составляет предмет диффузионной кинетики, в области которой применительно к рассматриваемым пористым электродам построено множество математических моделей, делающих попытку описать массотранпортные потери, и получены экспериментальные данные. С точки зрения современных требований к повышенной эффективности электродов особый интерес представляют электроды с малой удельной загрузкой платины $\left(0.1-0.3 \mathrm{mg} / \mathrm{cm}^{2}\right)$ и содержащие структурирующие добавки для снижения диффузионного сопротивления газам (в первую очередь кислороду). Так, в [1] представлена модель, описывающая межфазные транспортные потери (иономер, пленка воды, платиновые частицы) в диапазоне малых загрузок платины $\left(0.025-0.2 \mathrm{mg} / \mathrm{cm}^{2}\right)$ и данные эксперимента. В ряде работ [2-5] показано, что транспортные потери значительно возрастают при уменьшении загрузки платины. Для определения массотранспортных потерь в современных работах широко используется метод, основанный на измерении предельной диффузионной плотности тока. В [5] отмечается аномальное увеличение сопротивления транспорту кислорода на катоде при уменьшении удельной загрузки платины с 0.4 до $0.03 \mathrm{mg} / \mathrm{cm}^{2}$, причем авторы не находят объяснения этому эффекту. Авторы [6] связывают это с уменьшением электрохимически активной поверхности платины и увеличением потока кислорода к этой поверхности через пленку Nafion. Общим выводом в $[7,8]$ является наличие существенного вклада локального сопротивления транспорту кислорода на границах раздела фаз платина/Nafion в общее диффузионное сопротивление.

Исследование диффузионных характеристик разрабатываемых новых электродных материалов является важной задачей развития фундаментальных знаний о влиянии состава, структуры, технологии на особенности массового транспорта в пористых электродах. Авторы работ $[9,10]$ исследовали связь между составом катода водородного топливного элемента с протонпроводящей мембраной, пористостью и диффузионными свойствами при введении в него функциональных добавок гидрофобизатора и углеродных нанотрубок.

Наибольшее распространение при экспериментальном исследовании массотранспортных потерь получили два подхода, основанные на сравнении напряжения элемента во время работы с воздушно-гелиевой средой $(79 \% \mathrm{He}$, $\left.21 \% \mathrm{O}_{2}\right)$ или чистым $\mathrm{O}_{2}$ [11-13], и метод, основанный на измерении плотности предельного диффузионного тока $[2,14,15]$. В первом подходе использование коротких промежутков времени (2s) позволяет исключить влияние высыхания или изменение степени окисле- 
ния поверхности катализатора, связанные с непрерывной работой с этими газами, однако получаемые при использовании этого метода результаты не отражают диффузионные свойства в процессе функционирования электрода. Второй подход позволяет получать данные о диффузионных свойствах электрохимической системы при реальных и различных условиях функционирования (влажность газов, давление газов, условия продувки анода и др.).

Материалы пористых электродов, содержащих протонпроводящий иономер типа Nafion, наночастицы платины и углеродные наноматериалы (углеродная сажа, углеродные нановолокна), содержат большое число пор различного размера, большая доля которых является макропорами микронного размера, выполняющими функцию транспортных пор. Nafion в таких структурах, по крайней мере частично, покрывает поверхность платины. Благодаря гидрофильным свойствам Nafion способствует удержанию воды, выделяющейся в процессе работы катода, в результате чего происходит заполнение части пор водой. Массоперенос кислорода в такой системе осуществляется посредством свободномолекулярной и кнудсеновской диффузии в порах различного размера, а также посредством диффузии в жидкой воде и в пленке Nafion, покрывающей платину $[9,10]$. Общее сопротивление массопереносу в такой структуре меняется в зависимости от ее состояния, которое в значительной степени определяется водным обменом с окружающей средой. Водный же обмен с окружающей средой зависит как от свойств среды, так и от плотности тока мембранноэлектродного блока [16]. Таким образом, сопротивление массовому транспорту рассматриваемых структур переменно и зависит от условий функционирования. В связи с этим для измерения массотранспортных потерь использован подход, основанный на измерении плотности предельного диффузионного тока.

\section{1. Теоретические основы и использованные подходы}

Согласно современным представлениям диффузионной кинетики, перенос вещества (массовый транспорт) в электрохимической системе в общем случае осуществляется по трем механизмам, выражения (1), (2).

$$
\begin{gathered}
J_{\Sigma}=J_{\text {diffusion }}+J_{\text {migration }}+J_{\text {convection }} \\
J_{\Sigma}=-D_{i} \operatorname{grad} C_{i}-\left(z_{i} /\left|z_{i}\right|\right) u_{i} C_{i} \operatorname{grad} \varphi+C_{i} w,
\end{gathered}
$$

где $J_{\Sigma}$ и $J$ с индексами - суммарный массовый поток вещества и потоки вещества, переносимые по соответствующим механизмам (диффузия, миграция, конвекция), $z_{i}$ - заряд $i$-го компонента, $u_{i}-$ подвижность $i$-го компонента, $D_{i}$ - коэффициент диффузии, $\varphi-$ напряженность электрического поля между электродами, $w$ - скорость движения жидкости как целого, $C_{i}-$ концентрация $i$-го компонента.

\section{1. Упрощающие допущения}

Поскольку в нашем случае речь идет о переносе нейтральных молекул кислорода в пористом электроде с размерами пор микронного и субмикронного диапазона, не допускающими заметной конвекции, можно принять, что массоперенос осуществляется диффузией.

Второе допущение - согласно геометрии характерного электрода, его площадь $\left(\mathrm{cm}^{2}\right)$ существенно больше толщины (микроны) и диффузию можно свести к одномерной диффузии вдоль оси $X$, перпендикулярной к поверхности электрода.

Третьим допущением является принятие линейности профиля концентрации кислорода вдоль $X$.

В таком случае массоперенос можно описать уравнением Фика для одномерной стационарной диффузии (3)

$$
\partial C_{i} / \partial t=D_{i} \partial^{2} C_{i} / \partial x^{2}
$$

При рассмотрении простейшего случая стационарной диффузии $\partial C_{i} / \partial t=0$ граничные условия: при $x=0$ концентрация равна $C(0)$; при $x=\delta$ (толщина диффузионного слоя Нернста) концентрация равна $C^{*}$. Уравнение Фика для стационарной диффузии выглядит так:

$$
D_{i} \partial^{2} C_{i} / \partial x^{2}=0
$$

Решение этого уравнения

$$
\partial C_{i} / \partial x=-D_{i}\left[C^{*}-C(0)\right] / \delta=\text { const. }
$$

Отношение коэффициента диффузии к толщине диффузионного слоя Нернста $\left(m_{i}=D_{i} / \delta\right)$, принято называть коэффициентом массопереноса $(\mathrm{cm} / \mathrm{c})$. Величину, обратную коэффициенту массопереноса, обозначим как $R_{\text {Мт }}(\mathrm{c} / \mathrm{cm})$ - сопротивление массовому транспорту.

При протекании реакции восстановления кислорода, левая часть выражения (5) представляет собой плотность потока кислорода, и тогда его можно записать так

$$
J=-D\left[C^{*}-C(0)\right] / \delta,
$$

или

$$
J=\Delta C / R_{\mathrm{MT}} .
$$

Таким образом, исходя из значений плотности потока кислорода, который прямо связан с плотностью тока, и разности концентраций кислорода, можно вычислить сопротивление массовому транспорту (8)

$$
R_{\mathrm{MT}}=\Delta C / J
$$

Как указано в $[14,17]$, в случае постоянного $R_{\mathrm{MT}}$ во всей активной области падение концентрации кислорода связано лишь с диффузией (незначительный перепад давления), и тогда среднюю движущую силу диффузии $\Delta C$ можно вычислить, исходя из $\Delta C$ кислорода на входе ячейки $\left(\Delta C_{\text {in }}\right)$ и на выходе $\left(\Delta C_{\text {out }}\right)$, из соотношений $(9)-(12)$. Поскольку концентрацию кислорода в электроде в случае предельного диффузионного тока можно принять равной нулю, разность концентраций 
кислорода в объеме газа и электроде на входе и выходе сведется просто к концентрациям на входе и выходе

$$
\begin{gathered}
\Delta C=\left(C_{\text {in }}-C_{\text {out }}\right) / \ln \left(C_{\text {in }} / C_{\text {out }}\right), \\
C_{\text {in }}=y_{\text {in }} P_{\text {in }} / R T, \\
C_{\text {out }}=\left(Q_{\text {in }} C_{\text {in }}-J S\right) / Q_{\text {out }}, \\
Q_{\text {out }}=\left(Q_{\text {in }} P_{\text {in }}\right) / P_{\text {out }}-(R T J S) / P_{\text {out }},
\end{gathered}
$$

где $y_{i n}-$ мольная доля кислорода на входе, $P_{\text {in }}$ - давление на входе, $R$ - универсальная газовая постоянная, $T$ - температура, $Q_{\text {in }}$ и $Q_{\text {out }}$ - объемный поток газа на входе и выходе соответственно, $J$ - плотность диффузионного потока кислорода, расходуемого на катоде, $S$ - площадь видимой поверхности катода.

Плотность диффузионного потока кислорода связана с плотностью тока выражением

$$
J=j /(n F),
$$

где $j$ - плотность тока, $n-$ число электронов в реакции, $F-$ постоянная Фарадея.

Как видно из выражений (8)-(12), результаты вычисления $R_{\mathrm{MT}}$ зависят от плотности предельного тока и движущей силы диффузии (разности концентрации кислорода) (9).

Плотность измеренного предельного тока, полученного из экспериментальной поляризационной кривой (BAX) можно выразить соотношением (14)

$$
1 / j=1 / j_{\text {ext }}+1 / j_{\text {int }}+1 / j_{\text {reac }},
$$

где $j$ - плотность измеренного тока, $j_{\mathrm{ext}}-$ плотность внешнедиффузионного тока (из объема газа к электроду), $j_{\text {int }}$ - плотность внутридиффузионного тока (в пористом электроде), $j_{\text {reac }}$ - плотность тока электрохимической реакции восстановления кислорода.

Поскольку предельный ток наблюдается в области потенциалов, соответствующей большой поляризации, плотность тока реакции существенно больше плотностей диффузионных токов, и выполняются условия

$$
\begin{gathered}
j_{\text {reac }} \gg j_{\text {ext }}, \\
j_{\text {reac }} \gg j_{\text {int }}, \\
1 / \text { reac } \rightarrow 0 .
\end{gathered}
$$

И выражение (14) сводится к (18)

$$
1 / j=1 / j_{\text {ext }}+1 / j_{\text {int }} .
$$

Таким образом, стоит задача разделения плотностей внешнедиффузионного и внутридиффузионного токов.

В случае исследования катода в составе МЭБ снятие внешнедиффузионных ограничений возможно путем увеличения объемного расхода газа. При относительно небольших объемных потоках газа, когда его состав сильно меняется от входа к выходу за счет катодной реакции, влияние неточности поддержания потока газа на результат велико. С целью минимизации погрешности

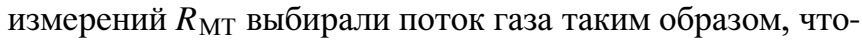
бы концентрация кислорода после контакта с катодом менялась незначительно, и движущая сила диффузии кислорода $\Delta C$ практически не зависела от колебания объемного потока газа.

В случае исследования материала на дисковом вращающемся электроде (ДВЭ), плотность внешнедиффузионного тока поддается вычислению по известным соотношениям Коутецкого-Левича.

Для вычисления движущей силы диффузии и плотности внутридиффузионного тока в случае ДВЭ необходимо провести серию измерений предельного тока при различных скоростях вращения электрода и построить зависимость в координатах Левича: $1 /(1 / \sqrt{\omega})$, где $\omega-$ угловая скорость вращения ДВЭ. При экстраполяции обратной плотности тока на бесконечную скорость вращения снимаются внешнедиффузионные ограничения:

$$
\begin{aligned}
& j_{\text {ext }} \gg j_{\text {int }}, \\
& 1 / j_{\text {ext }} \rightarrow 0 .
\end{aligned}
$$

И выражение (14) сводится к (21)

$$
1 / j=1 / j_{\text {int }} \text {. }
$$

Таким образом, отсечка по оси ординат соответствует обратной плотности внутридиффузионного тока при движущей силе, равной концентрации растворенного кислорода.

\section{2. Эксперимент}

Метод использовали для исследования массотранспортных потерь катода в составе МЭБ и электродного материала на дисковом вращающемся электроде.

\section{1. Мембранно-электродный блок}

При исследовании катода МЭБ использовали стандартную электрохимическую ячейку фирмы

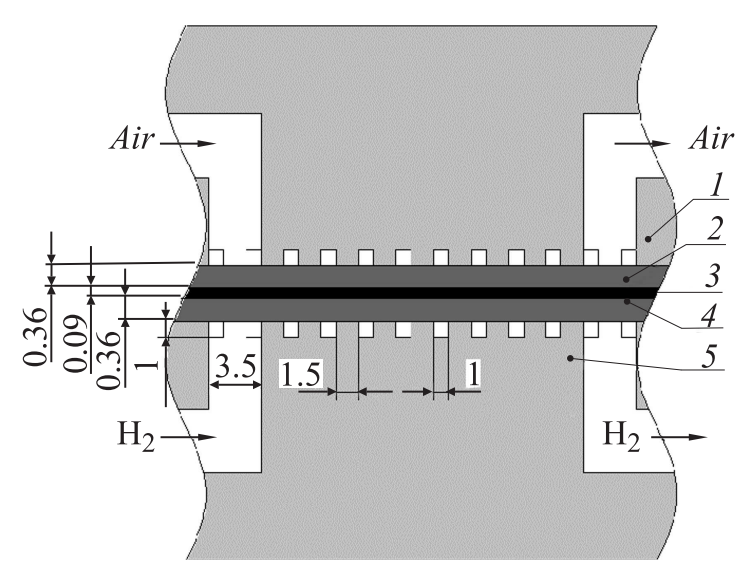

Рис. 1. Схема измерительной ячейки с закрытым катодным пространством: 1 - катодная часть корпуса ячейки; 2, 4 - газодиффузионные слои - пористая углеродная бумага, 3 - измеряемый МЭБ, 5 - анодная часть корпуса ячейки. 
Массотранспортные характеристики катодов, МЭБ и ДВЭ

\begin{tabular}{c|c|c|c|c|c|c|c|c}
\hline Объект & $\begin{array}{c}S_{\text {Pt, }} \\
\mathrm{cm}^{2}\end{array}$ & $\begin{array}{c}G_{\mathrm{Pt}}, \\
\mathrm{mg} / \mathrm{cm}^{2}\end{array}$ & $\begin{array}{c}j, \\
\mathrm{~mA} / \mathrm{cm}^{2}\end{array}$ & $\begin{array}{c}J \cdot 10^{8}, \\
\mathrm{~mol} / \mathrm{cm}^{2} \cdot \mathrm{c}\end{array}$ & $\begin{array}{c}C_{\text {in }} \cdot 10^{8}, \\
\mathrm{~mol} / \mathrm{cm}^{3}\end{array}$ & $\begin{array}{c}C_{\text {out }} \cdot 10^{8}, \\
\mathrm{~mol} / \mathrm{cm}^{3}\end{array}$ & $\begin{array}{c}\Delta \mathrm{CO}_{2} \cdot 10^{8} \\
\mathrm{~mol} / \mathrm{cm}^{3}\end{array}$ & $\begin{array}{c}R_{\mathrm{TM}} \\
\mathrm{c} / \mathrm{cm}^{3}\end{array}$ \\
\hline МЭБ & 90 & 0.15 & 600 & 155 & 818 & 802 & 810 & 5.23 \\
ДВЭ & 7.28 & 0.17 & 13.7 & 3.55 & 28.3 & 28.3 & 28.3 & 7.97
\end{tabular}

ElectroChem, Inc.'s типа FC-05-02 с контроллером температуры ТРМ 10; диапазон поддержания температуры в рабочем объеме от 20 до $180^{\circ} \mathrm{C}$, точность поддержания температуры $\pm 1^{\circ} \mathrm{C}$; избыточное давление газов до $2 \mathrm{~atm}$; балластное сопротивление менее $10 \mathrm{~m} \Omega$. Схематически ячейка представлена на рис. 1.

В качестве газодиффузионных слоев (ГДС) использовали углеродную бумагу типа Toray 90 (толщина $270 \mu \mathrm{m})$. Объемный поток воздуха на входе в ячейку $Q_{\text {in,air }}=560 \pm 10 \mathrm{~cm}^{3} / \mathrm{min} ; 9.33 \cdot 10^{-6} \mathrm{~m}^{3} / \mathrm{c}$. Объемный поток водорода на входе в ячейку $Q_{\mathrm{H}_{2}}=200 \pm 10 \mathrm{~cm}^{3} / \mathrm{min} ; 6.67 \cdot 10^{-6} \mathrm{~m}^{3} / \mathrm{c}$. Относительная влажность газов близка к $100 \%\left(0.023 \mathrm{~kg} / \mathrm{m}^{3}\right)$; температура $T=298 \mathrm{~K}$; давление $P=101325 \mathrm{~Pa}$, электроды работают на продув. Использовали симметричные МЭБ с одинаковыми катодом и анодом видимой площадью $1 \times 1 \mathrm{~cm}^{2}$.

После регистрации вольт-амперной характеристики (BАХ) МЭБ для вычисления плотности предельного тока выполняли аппроксимирование ВАХ эмпирическим соотношением, как в [14]

$$
E=E_{0}-b \lg i-R i+B \ln \left(1-i / i_{\lim }\right),
$$

где $E_{0}$ - напряжение разомкнутой цепи, $b$ - тафелевский наклон, $B$ - эмпирический коэффициент, $i_{\lim }-$ плотность предельного тока.

\section{2. Дисковый вращающийся электрод}

Электродный материал наносили на дисковый электрод с поверхностью из стеклоуглерода площадью $0.07 \mathrm{~cm}^{2}$. Для этого дисперсию каталитических чернил нужного состава в водно-изопропанольной смеси наносили на поверхность электрода и высушивали.

Поляризационные кривые (BAX) регистрировали на установке типа ВЭД-06 с потенциостатом IPC Pro. Измерения проводили в растворе $0.5 \mathrm{M}$ серной кислоты, находящемся в равновесии с воздухом или кислородом при температуре $25^{\circ} \mathrm{C}$. Потенциал рабочего электрода измеряли относительно хлорсеребряного электрода сравнения. Электрод вращали со скоростями в диапазоне 1000-6000 $\mathrm{min}^{-1}$.

На рис. 2 представлена схема диффузии кислорода в электрохимической ячейке. По оси ординат отложена концентрация молекулярного кислорода, по оси абсцисс - расстояние. Цифрами обозначены характерные области ячейки. Как видно из схемы, на результат измерений оказывает влияние внешняя диффузия (области 1 и 2), которое заключается в увеличении диффузионного

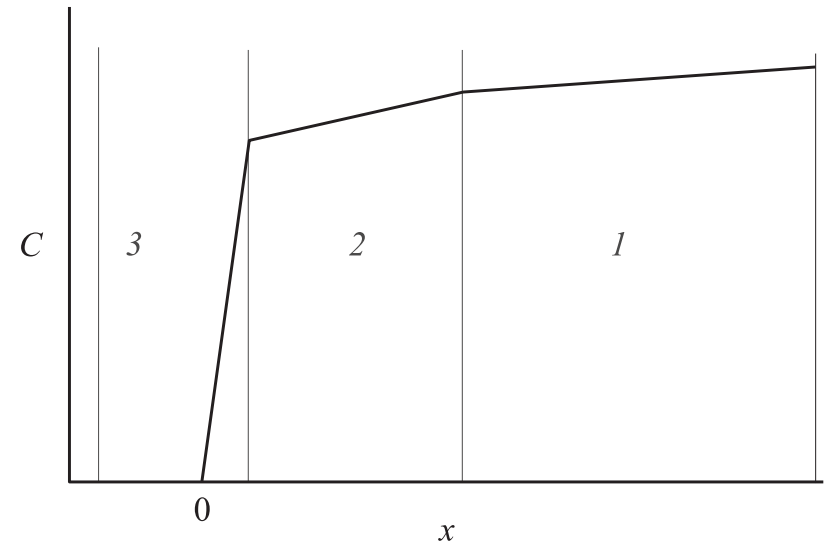

Рис. 2. Схематическое изображение диффузии кислорода в электрохимической измерительной ячейке; 1 - газоподводящий канал, 2 - газодиффузионный слой, 3 - пористый катод; сплошная линия соответствует концентрационному профилю молекулярного кислорода в каждой области ячейки.

сопротивления катода. С целью устранения этого влияния были проведены измерения с различным объемным потоком газа. Поток газа увеличивали до стабилизации плотности предельного тока. Отметим, что изменение толщины ГДС в таких условиях не приводило к заметному изменению предельной плотности тока. На основании этого сделан вывод о том, что внешнедиффузионное сопротивление массопереносу практически не проявляло себя и измеренную плотность предельного тока можно считать плотностью предельного тока внутренней диффузии, соответствующей $R_{\mathrm{MT}}$ катода. Движущая сила диффузии в этом случае равна концентрации кислорода в газе. При вычислении этой концентрации учитывали влажность газа.

\section{3. Результаты}

Метод был использован на примере образца НАНГ-68. Электродный материал имел состав: $(\mathrm{Pt} / \mathrm{C}(40 \% \mathrm{Pt})-$ $20 \%$, Таунит МД - 20\%, Nafion - 60\%). На основе этого электродного материала был изготовлен МЭБ и часть материала нанесена на ДВЭ.

На рис. 3 приведена характерная поляризационная кривая МЭБ. Штриховой линией на рисунке показана подгоночная кривая, полученная по соотношению (22).

На рис. 4 представлена в координатах Левича кинетическая кривая образца электродного материала на ДВЭ, полученная при скорости вращения электрода в 


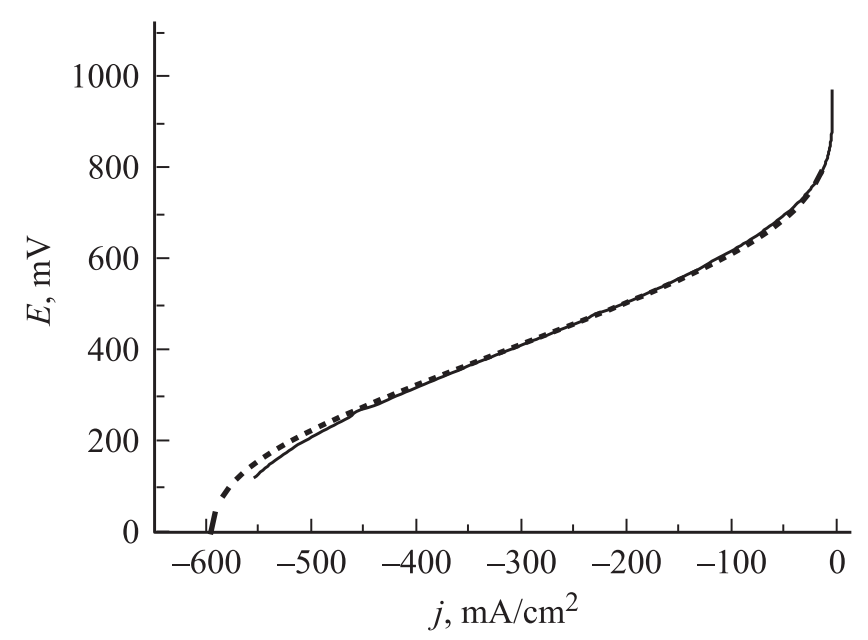

Рис. 3. Поляризационная (сплошная) и подгоночная (штриховая) кривые МЭБ НАНГ-68, пористость 61\%, загрузка Pt $0.15 \mathrm{mg} / \mathrm{cm}^{2}$, толщина $21 \mu \mathrm{m}$.

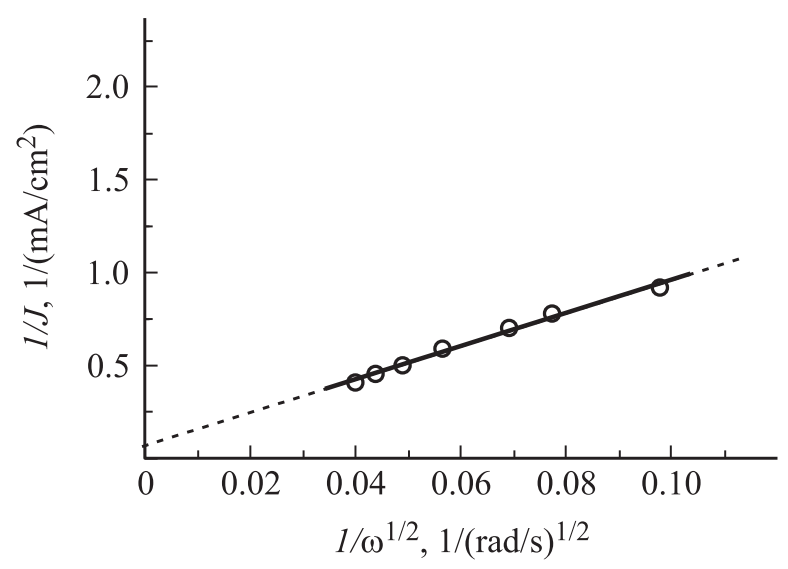

Pис. 4. Диффузионная кривая НАНГ-68 на дисковом вращающемся электроде.

диапазоне 1000-6000 $\mathrm{min}^{-1}$. Сплошная прямая аппроксимирует экспериментальные точки, штрихом показана экстраполяция на бесконечную скорость вращения ДВЭ (пересечение с осью ординат).

В таблице сведены данные вычисления массотранспортного сопротивления одного и того же электродного материала в двух реализациях - в составе МЭБ и на ДВЭ.

Следует отметить факт близости концентраций кислорода на входе и выходе, о чем говорилось выше. Как видно из таблицы, значения сопротивления массовому транспорту кислорода для одного и того же электродного материала в составе МЭБ и на ДВЭ довольно близки. Поскольку измерения на ДВЭ производили в водном растворе, т.е. поры электрода были заполнены раствором, близкие значения $R_{\text {Мт }}$ для катода МЭБ и образца на ДВЭ могут свидетельствовать о том, что при измерении предельного тока МЭБ также происходило заполнение пор катода водой.
При изменении толщины электрода в диапазоне $1-30 \mu \mathrm{m}$ и соответственно загрузки платины в ряде случаев не удавалось достичь режима диффузионного контроля катодного процесса, т. е. производная $d j / d E$ в конце поляризационной кривой не достигала значения, близкого к нулю. При этом следует отметить, что характер поляризационной кривой зависит от многих факторов, таких, как электродная активность, пористость, гидрофильность, состав газовой среды, толщина и др., поэтому строгой зависимости возможности достижения предельной плотности тока только от толщины не наблюдалось. Для каждого электродного материала необходимо подбирать толщину, исходя из критерия $d j / d E \approx 0$.

\section{Выводы}

1. Развит метод измерения диффузионного сопротивления структур электродных материалов пористых электродов на основе протонпроводящего иономера Nafion и углеродных наноматериалов со сверхвысокой пористостью, основанный на измерении плотности предельного тока.

2. С целью стабилизации условий измерения минимизирования влияния газоподводящих каналов и получения сопоставимых данных при исследовании различных образцов, разность концентраций кислорода на входе и выходе из электрохимической ячейки была приближена к нулю, измерения проводили при большом избытке кислорода. Это особенно важно для снятия внешнедиффузионных ограничений в случае катода с высокой пористостью и небольшим диффузионным сопротивлением.

3. Метод позволяет исследовать сопротивление массовому транспорту как катода МЭБ, так и электродного материала на ДВЭ.

4. Метод имеет прикладное значение при разработке новых электродных материалов и электродов с улучшенными характеристиками.

\section{Благодарности}

Экспериментальные исследования выполнены при частичной финансовой поддержке программ РФФИ № 1608-00797.

\section{Список литературы}

[1] Hao L., Moriyama K., Gu W., Wang C.-Y. // J. Electrochem. Soc. 2015. Vol. 162. N 8. P. F854-F867.

[2] Baker D.R., Caulk D.A., Neyerlin K.C., Murphy M.W. // J. Electrochem. Soc. 2009. Vol. 156. N 9. P. B991-B1003.

[3] Nonoyama N., Okazaki S., Weber A.Z., Ikogi Y., Yoshida T. // J. Electrochem. Soc. 2011. Vol. 158. P. B416-23.

[4] Reshetenko T., Kulikovsky A. // J. Electrochem. Soc. 2016. Vol. 163. N 3. P. F238-F246.

[5] Greszler T.A., Caulk D., Sinha P. // J. Electrochem. Soc. 2012. Vol. 159. P. F831-840. 
[6] Weber A.Z., Borup R.L., Darling R.M., Das P.K., Dursch T.J., Gu W., Harvey D., Kusoglu A., Litster S., Mench M.M., Mukundan R., Owejan J.P., Pharoah J.G., Secanell M., Zenyuk I.V. // J. Electrochem. Soc. 2014. Vol. 161. P. F1254-1299.

[7] Ono Y., Mashio T., Takaichi S., Ohma A., Kanesaka H., Shinohara K. // ECS Transactions. 2010. Vol. 28. P. 69-78.

[8] Fukuyama Y., Shiomi T., Kotaka T., Tabuchi Y. // Electrochim. Acta. 2014. Vol. 117. P. 367-378.

[9] Глебова Н.В., Нечитайлов А.А., Краснова А.О., Томасов А.А., Зеленина Н.К. // ЖПХ. 2015. Т. 88. Вып. 5. C. 726-731. (Glebova N.V., Nechitailov A.A., Krasnova A.O., Tomasov A.A., Zelenina N.K. // Russ. J. Appl. Chem. 2015. Vol. 88. N 5. P. 769-774.)

[10] Нечитайлов А.А., Глебова Н.В., Краснова А.О., Томасов А.А., Зеленина Н.К. // ЖТФ. 2015. Т. 85. Вып. 11. C. 97-103. (Nechitailov A.A., Glebova N.V., Krasnova A.O., Tomasov A.A., Zelenina N.K. // Tech. Phys. 2015. Vol. 60. N 11. P. 1670-1676.)

[11] Boillat P., Oberholzer P., Kaestner A., Siegrist R., Lehmann E.H., Scherer G.G., Wokau A. // J. Electrochem. Soc. 2012. Vol. 159. P. F210-F218.

[12] Biesdorf J., Forner-Cuenca A., Schmidt T. J., Boillat P. // J. Electrochem. Soc. 2015. Vol. 162. N 10. P. F1243-F1252.

[13] Forner-Cuenca A., Biesdorf J., Manzi-Orezzoli V., Gubler L., Schmidt T.J., Boillat. P. // J. Electrochem. Soc. Vol. 163. N 13. P. F1389-F1398.

[14] Beuscher U. // J. Electrochem. Soc. Vol. 153. N 9. P. A1788A1793.

[15] Hayashi T., Tabe Y., Chikahisa T. // ECS Trans. 2016. Vol. 75. N 14. P. 373-383.

[16] Нечитайлов А.А., Глебова Н.В., Кочкина Д.В., Томасов А.А., Зеленина Н.К., Терукова Е.Е. // Письма в ЖТФ. 2013. Т. 39. Вып. 7. С. 15-26.

[17] Cussler E.L. Diffusion-Mass Transfer in Fluid Systems. Cambridge, UK, Cambridge University Press. 1997. 2nd Ed., $79 \mathrm{p}$. 